\title{
La fertilité chez le blessé médullaire. Effet du niveau de la lésion médullaire
}

\author{
P. DENYS, A. EVEN SCHNEIDER, J.M. SOLER, D. BEN SMAIL, E. CHARTIER-KASTLER \\ Service de rééducation Neurologique, Hôpital Raymond Poincaré, Garches
}

\section{RÉSUMÉ}

Les blessés médullaires souffrent d'anéjaculation dans une grande majorité des cas ainsi que d'anomalies de la qualité du sperme qui altèrent leur fertilité. La prise en charge doit prendre en compte les comorbidités que sont la spasticité, les troubles urinaires et la fonction anorectale qui modifient la fonction sexuelle. Le niveau de la lésion médullaire ainsi que son étendue ont un impact à la fois sur la possibilité d'obtention d'une éjaculation réflexe et sur la qualité du sperme.

Mots clés : Blessés médullaires, fertilité, anéjaculation, vessie neurologique, niveau lésionnel.

\section{INTRODUCTION}

Dans l'inconscient collectif il est souvent rencontré l'opinion suivante : une personne handicapée est une personne asexuée, un homme en fauteuil roulant est impuissant et stérile. Heureusement le développement de la formation des médecins sur ce sujet, une recherche de qualité sur la neurophysiologie de la fonction sexuelle, les progrès de la thérapeutique ont modifié la prise en charge de ces patients. Un homme blessé médullaire n'est pas un être asexué et l'on peut même dire avec certitude que la prise en charge des troubles sexuels améliore les conditions de la réinsertion et la qualité de vie de ces patients [1].

La prise en charge des troubles sexuels et de la fertilité des hommes paraplégiques a plusieurs spécificités que nous aborderons successivement, les aspects généraux de la neurophysiologie de l'éjaculation, les conditions générales de la prise en charge et en particulier des comorbidités présentes sur la fonction vésicosphinctérienne et anorectale, les effets du niveau de la lésion médullaire et en particulier du syndrome lésionnel. Par ailleurs il est important de comprendre que la fertilité de l'homme paraplégique est altérée par deux éléments différents que sont la capacité à éjaculer et secondairement la qualité du sperme.

\section{LA NEUROPHYSIOLOGIE DE L'ÉJACULATION}

L'éjaculation est sous dépendance de trois centres médullaires $[2,3]$

Le centre sympathique D10 L2 adresse par l'intermédiaire du nerf hypogastrique ses fibres post ganglionnaires aux vésicules séminales, aux canaux déférents dont il assure le péristaltisme, les fibres musculaires lisses de la prostate et enfin il assure la clôture du col vésical. On comprend alors aisément qu'il est responsable de la première partie de la réponse éjaculatoire qui est l'émission du sperme

Le centre parasympathique sacré S2 S4 assure une partie de l'innervation prostatique et assure probablement une partie de la sécrétion prostatique.

Le centre somatique sacré S2 S4 assure par l'intermédiaire du nerf pudendal l'innervation des muscles bulbo spongieux et ischiocaverneux qui assurent avec les autres muscles du plancher périnéal les contractions cloniques qui entraînent la sortie de l'éjaculat vers l'extérieur. Il s'agit de la deuxième partie de l'éjaculation.

Face à la complexité de la régulation neurologique de l'éjaculation on comprend aisément qu'une atteinte neuro-

\section{Correspondance :}

Pierre DENYS, Service de rééducation Neurologique, Hôpital Raymond Poincaré, 92380 Garches

Communication à la Session SALF-AFU des $6^{\circ}$ Journées de la Fédération Française d'Étude de la Reproduction, Lyon, 5-7 septembre 2001 
logique médullaire puisse facilement altérer cette physiologie que ce soit par la disparition du contrôle volontaire, par l'absence de coordination entre les centres médullaires et enfin selon les niveaux s'il existe une destruction des centres médullaires évaluée par l'étude du syndrome lésionnel.

\section{LES COMORBIDITÉS}

Il est important de comprendre que la pathologie médullaire entraîne une comorbidité importante qu'il n'est pas possible de dissocier de la prise en charge des troubles génitosexuels. Les comorbidités principales sont les troubles urinaires, les troubles anorectaux, la spasticité.

\section{Les troubles urinaires}

Tous les patients atteints d'une pathologie médullaire souffrent de troubles urinaires. Selon le niveau et le caractère complet de la lésion ces troubles sont plus ou moins importants. Dans la grande majorité il s'agit d'une hyperactivité de vessie avec une dyssynergie vésicosphinctérienne. Les conséquences sont moins importantes en terme de mortalité qu'il y a 20 ans ou les troubles urinaires représentaient la première cause de mortalité. Il n'en reste pas moins vrai que malgré les progrès des thérapeutiques chirurgicales et médicales, ces troubles urinaires restent la première cause de morbidité [4] et en particulier par l'intermédiaire des infections urinaires. En France, dans la majorité des équipes qui prennent en charge ces patients, le choix therapeutique privilégié comporte l'association d'autosondages propres intermittents et de parasympathicolytiques. Cette association permet la restauration d'une continence, la maitrise des facteurs de risques de dégradation rénale par la diminution des pressions intravésicales ainsi qu'une vidange complète et régulière de la vessie pour contrôler au mieux les infections urinaires. Il est bien entendu évident que la continence est un préalable indispensable à la restauration de la fonction sexuelle. Il n'en reste pas moins vrai que les infections urinaires subsistent souvent malgré le respect des conditions d'efficacité de ce choix thérapeutique et qu'elles représentent un vrai challenge pour la fertilité [5]. Il semble par ailleurs que la qualité du sperme soit différente selon le type de prise en charge [6] et que les meilleures qualités soient rencontrées chez les patients sous autosondage [7,8]. Enfin les pressions mictionelles élevées chez les patients urinant par mictions réflexes altèrent la mobilité des spermatozoïdes. En revanche la présence dans les urines d'infections urinaires n'est pas prédictive de qualité altérée du sperme [6]. Il est donc logique de démarrer la prise en charge de ces patients par l'équilibration des troubles urinaires.

\section{Les troubles anorectaux}

De même que pour la vessie et la fonction génitosexuelle le contrôle volontaire de la défécation est altéré. Il faut s'as- surer que la continence anale est acquise souvent au prix de l'utilisation de techniques de défécations réflexes programmées qui permettent aussi de contrôler le risque toujours présent de constipation.

\section{La spasticité}

La neuroplasticité, la perte des influx supérieurs ainsi qu'une modification des propriétés du muscle strié peut entraîner une modification fonctionnellement gênante du tonus musculaire. Cette hypertonie ou ces spasmes peuvent être contrôlées pharmacologiquement par différentes drogues soit orales soit intrathécales. Le baclofen peut malheureusement avoir un effet délétère sur la possibilité de boucler un réflexe d'éjaculation [9]. Il convient de s'en assurer et en cas d'échec de la stimulation vibratoire de modifier les doses de baclofen pour permettre de boucler des éjaculations réflexes plus aisées.

\section{LES EFFETS DU NIVEAU DE LA LÉSION SUR LA FERTILITÉ}

Après une lésion de la moelle épinière on peut cliniquement facilement apprécier le niveau de la lésion, il est en revanche plus difficile d'évaluer l'étendue de la lésion médullaire où elle est afonctionelle. Il est en effet très différent d'avoir une lésion en T8 mais un syndrome lésionnel préservant D10 L2 et une autre lésion avec une destruction plus étendue de la moelle détruisant ce centre sympathique et donc rendant de manière quasi impossible l'émission de sperme. Malheureusement la grande majorité des études de la littérature ne s'intéressent qu'au niveau de la lésion sans tenir compte de l'étendue du syndrome lésionnel.

La possibilité d'obtenir une éjaculation est souvent altérée par la lésion médullaire. Celle ci n'est conservée en moyenne dans la littérature que chez environs $15 \%$ des patients [2]. Concernant la capacité à obtenir une éjaculation réflexe Brindley dans les premières grandes séries de la littérature notaient que la réponse à la vibration était corrélée à la présence d'une flexion de hanche [10]. Mais il faut pondérer ce résultat par le fait que $25 \%$ des patients qui conservaient une flexion de hanche ne pouvaient avoir une éjaculation à la stimulation vibratoire. Chapelle [11] notait que l'existence d'un syndrome lésionnel incluant D10 L2 entraînait une réponse absente à l'utilisation d'inhibiteurs de l'acetyl choline estérase dans une population homogène avec des lésions cliniquement complètes. Enfin plus récemment Sonksen [12] lui notait que l'éjaculation antégrade n'est présente qu'en cas de lésion supérieure à D10. Mais pour d'autres auteurs un réflexe bulbocaverneux est prédicteur de l'absence d'éjaculation au vibreur [13].

Concernant la qualité du sperme les données de la littérature retrouvent globalement un nombre de spermatozoïdes élevée avec une mobilité altérée $[14,15]$. Les causes en ont 
été longuement discutées que ce soit le mode de fonctionnement urinaire, les infections urinaires ou génitales, l'hyperthermie, la position assise en continu, les anomalies hormonales, les anticorps antispermatozoïdes et l'absence d'éjaculation. Pour ce qui concerne le niveau et le type de lésion seules les données de Chapelle donnent un éclairage. En cas de lésion incluant dans le syndrome lésionnel le centre sympathique, il a pu montrer que l'éjaculation était impossible à obtenir, que la taille du testicule était diminuée et enfin qu'à la biopsie on retrouvait une altération spécifique des tubes séminifères $[16,17]$. Si l'on comprend aisément que la destruction du centre sympathique ne permet pas l'émission de sperme, en revanche l'atteinte testiculaire est plus difficile à comprendre et il n'existe pas à ce jour de mécanisme clairement élucidé.

L'étude des niveaux lésionnels est intéressante pour étudier les mécanismes qui altèrent la fonction sexuelle chez les para ou tétraplégiques. Ces études n'ont de sens que si les lésions sont complètes et que l'on étudie de manière parallèle l'étendue du syndrome lésionnel. 50\% des patients sont donc exclus de ces séries puisqu'ils présentent des lésions médullaires incomplètes. Enfin il n'existe pas de possibilité clinique ou paraclinique d'affirmer le caractère parfaitement complet d'une lésion médullaire. Cela plaide pour une démarche thérapeutique pragmatique ou les différents moyens thérapeutiques qui permettent le traitement de l'anéjaculation doivent être essayés avant de se poser la question de la procréation médicalement assistée.

\section{REFERENCES}

1. WHITE M.J., et al. : Sexual activities, concerns and interests of men with spinal cord injury. Am. J. Phys. Med. Rehabil., 1992, 71 : 225-231.

2. BIERING-SORENSEN F., SONKSEN J. : Sexual function in spinal cord lesioned men. Spinal Cord., 2001, 39 : 455-470.

3. GIULIANO F., RAMPIN O. : Central neural regulation of penile erection. Neurosci. Biobehav. Rev., 2000, 24 : 517-533.

4. WHITENECK G.G., et al. : Mortality, morbidity, and psychosocial outcomes of persons spinal cord injured more than 20 years ago. Paraplegia, 1992, $30: 617-630$.

5. WELD K.J., DMOCHOWSKI R.R. : Association of level of injury and bladder behavior in patients with post-traumatic spinal cord injury. Urology, 2000, $55:$ 490-494.

6. OHL A., et al. : Fertility of spinal cord injured males: effect of genitourinary infection and bladder management on results of electroejaculation. J. Am. Paraplegia. Soc., 1992, 15 : 53-59.

7. OHL D.A., et al. : Predictors of success in electroejaculation of spinal cord injured men. J. Urol., 1989, 142 : 1483-1486.

8. RUTKOWSKI S.B., et al. : The influence of bladder management on fertility in spinal cord injured males. Paraplegia, 1995, $33: 263-266$.

9. DENYS P., et al. : Side effects of chronic intrathecal baclofen on erection and ejaculation in patients with spinal cord lesions. Arch.
Phys. Med. Rehabil., 1998, 79 : 494-496.

10. BRINDLEY G.S. : The fertility of men with spinal injuries. Paraplegia, 1984, 22 : 337-348.

11. CHAPELLE P.A., et al. : Treatment of anejaculation in the total paraplegic by subcutaneous injection of Physostigmine. Paraplegia, 1983, $21:$ 30-36.

12. SONKSEN J., BIERING-SORENSEN F, and KRISTENSEN J.K. : Ejaculation induced by penile vibratory stimulation in men with spinal cord injuries. The importance of the vibratory amplitude. Paraplegia, 1994, $32: 651-660$.

13. SZASZ G., CARPENTER C. : Clinical observations in vibratory stimulation of the penis of men with spinal cord injury. Arch. Sex. Behav., 1989, $18: 461-474$.

14. BRACKETT N.L., et al. : An analysis of 653 trials of penile vibratory stimulation in men with spinal cord injury, J. Urol., 1998, 159 : $1931-1934$.

15. SONKSEN J,, et al. : Quality of semen obtained by penile vibratory stimulation in men with spinal cord injuries: observations and predictors. Urology, $1996,48: 453-457$.

16. CHAPELLE P.A., et al. : Trophic effects on testes in paraplegics. Paraplegia, 1993, 31 : 576-583.

17. CHAPELLE P.A., et al. : Neurological correlations of ejaculation and testicular size in men with a complete spinal cord section. I. Neurol. Neurosurg. Psychiatry, 1988, 51 : 197-202.

\section{ABSTRACT}

\section{Fertility in Spinal cord injured males. Effect of the level of spinal lesion.}

\section{P. DENYS, A. EVEN SCHNEIDER, J.M. SOLER, D. BEN SMAIL, E. CHARTIER-KASTLER}

The majority of spinal cord injured males cannot procreate naturally due to anejaculation as well as abnormal sperm characteristics. Treatment of this impaired fertility must be associated with treatment of neurogenic urinary tract disorders, bowel dysfunction and spasticity. The level of the spinal lesion and the spinal cord injury syndrome influence the possibility of inducing reflex ejaculation by penile vibratory stimulation and sperm quality.

Key words: Spinal cord injury, fertility, anejaculation, neurogenic bladder, spinal level. 